\title{
JNK1-mediated phosphorylation of Smac/DIABLO at the serine 6 residue is functionally linked to its mitochondrial release during TNF- $\alpha$-induced apoptosis of HeLa cells
}

\author{
BYOUNGDUCK PARK \\ College of Pharmacy, Keimyung University, Daegu 704-701, Republic of Korea
}

Received January 24, 2014; Accepted June 20, 2014

DOI: $10.3892 / \mathrm{mmr} .2014 .2625$

\begin{abstract}
The second mitochondria-derived activator of caspases (Smac/DIABLO), is a mitochondrial protein that is released along with cytochrome $c$ during apoptosis and promotes caspase activity. It has been reported that c-Jun N-terminal kinase (JNK) is involved in the regulation of Smac release during apoptosis; however, the specific role of JNK is not well understood. The aim of the present study was to investigate whether JNK 1 is activated during apoptosis induced by tumor necrosis factor- $\alpha$ (TNF- $\alpha$ ) and whether the activation of JNK1 is functionally associated with Smac release from the mitochondria in HeLa cells. It was determined that during apoptotic progression induced by TNF- $\alpha$, JNK1 is activated and translocated into the mitochondria. It was also shown that Smac release is markedly promoted by transient expression of JNK1, while it is suppressed by the expression of a dominant negative version of JNK1. Furthermore, expression of JNK1 also increases the levels of TNF- $\alpha$-induced caspase- 3 activity. To determine whether JNK1 activity is directly involved in the release of Smac, an in vitro phosphorylation assay was performed with the N-terminal 10-mer peptide fragment of Smac (pep0110). The results indicated that pep0110 is phosphorylated in dose and time-dependent manners by active JNK1, and that the phosphorylation of Smac at the N-terminal serine 6 residue is functionally linked to Smac release during TNF- $\alpha$-induced apoptosis. In conclusion, this suggests that Smac is a major physiological substrate of JNK1 in the regulation of apoptosis.
\end{abstract}

\section{Introduction}

c-Jun N-terminal protein kinase (JNK) is a subfamily of the mitogen-activated protein kinase (MAPK) superfamily (1). JNK has two ubiquitously expressed isoforms, JNK1 and

Correspondence to: Dr Byoungduck Park, College of Pharmacy, Keimyung University, 1095 Dalgubeol-daero, Daegu 704-701, Republic of Korea

E-mail: bdpark@kmu.ac.kr; pduggiebbol@gmail.com

Key words: JNK1, Smac, mitochondria, phosphorylation, apoptosis
JNK2, and a tissue-specific isoform, JNK3; all of which have two different splicing forms (p54 and p46) (2). JNK is activated by sequential protein phosphorylation through a MAP kinase module in response to a variety of extracellular stimuli, including tumor necrosis factor- $\alpha(\mathrm{TNF}-\alpha)(3)$ and UV light (4). Activation of JNK is regulated by scaffold proteins, including JNK-interacting proteins, $\beta$-arrestin, and nuclear factor of $\kappa$ light polypeptide gene enhancer in B-cells $(\mathrm{NF}-\kappa \mathrm{B})(5)$. JNK regulates multiple cellular activities, ranging from gene expression to apoptosis, and there is evidence that JNK also contributes to cell survival. Genetic evidence from a previous study reveals that JNK1 and JNK2 are involved in the survival of neuronal cells in mouse fore and hindbrain regions during development (5).

Activation of tumor necrosis factor receptor 1 (TNFR1) by TNF- $\alpha$ leads to the recruitment of the TNFRSF1A-associated via death domain (TRADD) protein, which in turn recruits Fas (TNFRSF1A)-associated via death domain, leading to caspase-8 activation and apoptosis via the extrinsic pathway (6). However, activation of TNF- $\alpha$ is not always cytotoxic to cells, as recruitment of TNF- $\alpha$ receptor associated factor 2 and receptor TNFRSF-interacting serine-threonine kinase by TRADD can activate $\mathrm{NF}-\kappa \mathrm{B}$ and $\mathrm{JNK}$, which have a pivotal role in cell proliferation and survival (7). The involvement of JNK in TNF- $\alpha$-mediated apoptosis is the subject of much debate (8). Inhibition of NF- $\kappa \mathrm{B}$ results in sustained activation of JNK, which may directly promote TNF- $\alpha$-mediated apoptosis (9). Despite the fact that sustained activation of JNK promotes cell death, the molecular mechanisms by which JNK contributes to TNF- $\alpha$-mediated apoptosis remain to be addressed.

The intrinsic apoptotic pathway is the result of activation of mitochondria-mediated cell death events, including changes in mitochondrial membrane permeability and the subsequent release of proapoptotic factors (10). Although the apoptotic pathway through death receptors and the pathway through mitochondria are capable of operating independently, previous studies suggest that a crosstalk exists between the two pathways (11).

The second mitochondria-derived activator of caspases (Smac/DIABLO) is a 29-kDa mitochondrial protein which, following an apoptotic trigger, is processed to a $23-\mathrm{kDa}$ mature protein and translocates to the cytosol (12). In addition to its interaction with X-linked inhibitor of apoptosis protein (XIAP), Smac/DIABLO has been shown to bind other inhibitor 
of apoptosis (IAP) proteins including c-IAP1, c-IAP2, survivin and baculoviral Op-IAP $(13,14)$. Mature Smac/DIABLO binds the BIR3 domain of XIAP with an N-terminus recognition motif similar to that which binds caspase-9. This same amino terminal sequence of Smac/DIABLO also forms a stable complex with the BIR2 domain of XIAP, meaning that Smac/DIABLO can act as a competitor in the XIAP-dependent inhibition of caspase- 3 and caspase-7 (12).

Previous studies have shown that JNK mediates the release of cytochrome $c$ (15) and Smac (16). Furthermore, JNK has been linked to apoptosis; specifically, two serine residues (Ser63 and 73) within c-Jun are direct targets for JNK (17). However, the mechanism by which Smac is released from the mitochondria has yet to be elucidated. The aim of the present study was to investigate whether JNK1 is activated during apoptosis induced by tumor necrosis factor- $\alpha$ (TNF- $\alpha$ ) and whether the activation of JNK1 is functionally associated with Smac release from the mitochondria in HeLa cells.

\section{Materials and methods}

Reagents. Cycloheximide was obtained from Sigma-Aldrich (St.Louis,MO,USA) and dissolved in phosphate-buffered saline (PBS) at a concentration of $5 \mathrm{mg} / \mathrm{ml}$. TNF- $\alpha$ was obtained from Biosource International Inc. (Camarillo, CA, USA). Caspase-3 substrate, Ac-Asp-Glu-Val-Asp-7-amino-4-methylcoumarin (Ac-DEVD-AMC), was purchased from BD Biosciences Pharmingen (San Diego, CA, USA).

Cell culture, apoptotic induction and transfection. HeLa cells (American Type Culture Collection, Manassas, VA, USA) were maintained at $37^{\circ} \mathrm{C}$ and $5 \% \mathrm{CO}_{2}$ in DMEM supplemented with $10 \%$ heated-inactivated FBS and antibiotics-antimycotics (all Gibco-BRL, Carlsbad, CA, USA). For apoptotic induction, cells were treated with $5 \mathrm{ng} / \mathrm{ml} \mathrm{TNF}-\alpha$ and $5 \mu \mathrm{g} / \mathrm{ml}$ cycloheximide. As TNF- $\alpha$ alone had no effect on the viability of the HeLa cells, the protein synthesis inhibitor cycloheximide was used in combination with TNF- $\alpha$ for the induction of apoptosis in HeLa cells. Transient transfection was performed in 100-mm culture plates using PolyFect (Qiagen, Valencia, CA, USA). Cells $\left(5 \times 10^{5}\right)$ were seeded and transfected using $10 \mu \mathrm{g}$ total DNA. The transfected cells were incubated for $24 \mathrm{~h}$ and subsequently treated for 0,3 or $6 \mathrm{~h}$ with TNF- $\alpha(5 \mathrm{ng} / \mathrm{ml})$ and cycloheximide $(5 \mu \mathrm{g} / \mathrm{ml})$ for subcellular fractionation, caspase-3 activity assay and western blotting.

Western blot analysis. Cell pellets were washed with PBS and lysed in lysis buffer $(0.5 \%$ Triton X-100, $20 \mathrm{mM}$ Tris, pH 7.5, $2 \mathrm{mM} \mathrm{MgCl}, 1 \mathrm{mM}$ dithiothreitol (DTT), $1 \mathrm{mM}$ EGTA, $50 \mathrm{mM} \beta$-glycerophosphate, $25 \mathrm{mM} \mathrm{NaF}, 1 \mathrm{mM}$ $\mathrm{Na}_{3} \mathrm{VO}_{4}, 100 \mu \mathrm{g} / \mathrm{ml}$ phenylmethanesulfonyl fluoride (PMSF), $10 \mathrm{mM}$ protease inhibitor cocktail in phosphate buffer, $\mathrm{pH}$ 7.0) on ice for $1 \mathrm{~h}$, then centrifuged at $15,000 \mathrm{x} \mathrm{g}$ for $20 \mathrm{~min}$ at $4^{\circ} \mathrm{C}$. Lysates were subjected to SDS-PAGE and transferred to a polyvinylidene difluoride membrane (Gibco-BRL). The membrane was blocked with 5\% non-fat dried milk in PBS $(137 \mathrm{mM} \mathrm{NaCl}, 2.7 \mathrm{mM} \mathrm{KCl}, 4.3 \mathrm{mM}$ $\mathrm{Na}_{2} \mathrm{HPO}_{4}-7 \mathrm{H}_{2} \mathrm{O}, 1.4 \mathrm{mM} \mathrm{KH} \mathrm{PO}_{4}$, pH 7.4) containing $0.05 \%$ Tween 20 and incubated in a 1:1,000 dilution of the following antibodies: Rabbit polyclonal anti-JNK1, rabbit polyclonal anti-poly ADP-ribose polymerase (PARP), rabbit polyclonal anti- $\beta$-actin, and rabbit polyclonal anti-cytochrome $c$ oxidase IV (COX IV) (Santa Cruz Biotechnology, Inc., Santa Cruz, CA, USA), mouse monoclonal anti-phospho-JNK, mouse monoclonal anti-Smac/DIABLO and mouse monoclonal anti-FLAG antibodies (Cell Signaling Technology, Inc., Beverly, MA, USA). Bands were visualized with horseradish peroxidase-conjugated antibodies (Pierce, Rockford, IL, USA) and the Enhanced Chemiluminescence system (Pierce, Rockford, IL, USA).

Preparation of cytosolic and mitochondrial fractions. The cell pellet was washed with PBS and was suspended in 2 volumes of buffer A (20 mM HEPES, pH 7.5, $1.5 \mathrm{mM}$ $\mathrm{MgCl}_{2}, 10 \mathrm{mM} \mathrm{KCl}, 1 \mathrm{mM}$ EGTA, $1 \mathrm{mM}$ DTT, $0.1 \mathrm{mM}$ PMSF, and $10 \mathrm{mM}$ protease inhibitor cocktail in phosphate buffer, $\mathrm{pH}$ 7.0) containing $250 \mathrm{nM}$ sucrose. The pellet was homogenized using a Dounce homogenizer (BBI-8530718, Thomas Scientific, Swedesboro, NJ, USA), centrifuged at $800 \mathrm{x}$ g for $10 \mathrm{~min}$ at $4^{\circ} \mathrm{C}$ and the supernatant was collected and further centrifuged at $100,000 \mathrm{x} \mathrm{g}$ for $1 \mathrm{~h}$ at $4^{\circ} \mathrm{C}$. The supernatant was used as cytosolic extract, and the cell pellet was lysed and used as the mitochondrial fraction

Caspase-3 activity assay. Cells were cultured in 100-mm dishes and treated with TNF- $\alpha$ and cycloheximide for the indicated time periods. Cell lysates were prepared in the lysis buffer (50 mM Tris- $\mathrm{HCl}, \mathrm{pH} 7.5,150 \mathrm{mM} \mathrm{NaCl}, 1 \mathrm{mM}$ EDTA, 1 mM EGTA, 1\% NP-40, 1 mM PMSF) supplemented with $10 \mathrm{mM}$ protease inhibitor cocktail in phosphate buffer, $\mathrm{pH}$ 7.0. The lysates were centrifuged at $10,000 \mathrm{x}$ g for $10 \mathrm{~min}$ and the supernatant was collected. Cell lysates were added to the reaction buffer (20 mM HEPES pH 7.5, 10\% glycerol, $2 \mathrm{mM}$ DTT) containing $25 \mu \mathrm{M}$ Ac-DEVD-AMC in 96-well plates. Lysates were incubated at $37^{\circ} \mathrm{C}$ for $1 \mathrm{~h}$. Fluorescence of the cleavage product was measured using a SpectraFluor F129003 (Tecan, Maenndorf, Switzerland) at excitation and emission wavelengths of $360 \mathrm{~nm}$ and $460 \mathrm{~nm}$, respectively

Peptide filter binding assay. The kinase reaction was performed as follows: Recombinant JNK1 was incubated with synthetic peptide in the presence of $5 \mathrm{X}$ reaction buffer (40 mMMOPS, pH 7.0, 1 mMEDTA), [32-P] ATP and distilled water. The reaction tube was placed in a microcentrifuge and a pulse-spin was used to wash the components into the base of the tube. The tube was incubated for $10 \mathrm{~min}$ at $30^{\circ} \mathrm{C}$ and the reactants subsequently transferred onto the center of P81 phosphocellulose paper (Millipore, Billerica, MA, USA) and washed 3 times with $0.75 \%$ phosphoric acid for $5 \mathrm{~min}$ at room temperature. The assay squares were washed once with acetone for $5 \mathrm{~min}$ at room temperature and transferred to scintillation vials, in which scintillation cocktail was added. The sample was read in a scintillation counter. Scintillation vials, cocktail and counter were purchased from PerkinElmer (Waltham, MA, USA).

\section{Results}

JNK1 is upregulated during TNF- $\alpha$-induced apoptosis in HeL a cells. To examine whether JNK1 activity is upregulated 
A

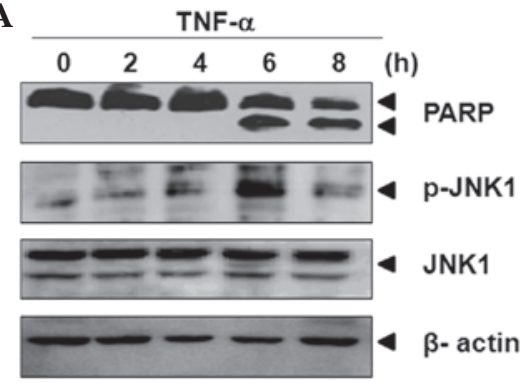

B

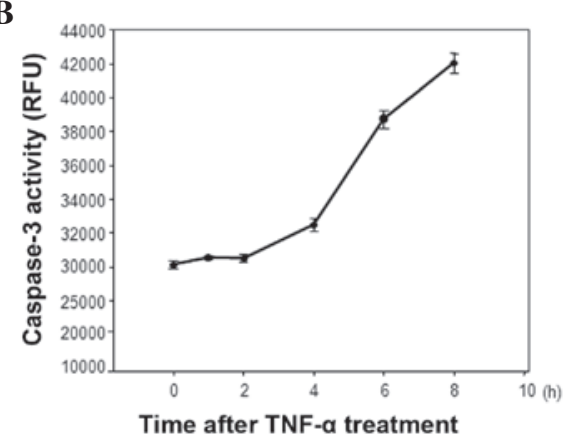

Figure 1. c-Jun N-terminal protein kinase 1 (JNK1) activity is upregulated during tumour necrosis factor- $\alpha$ (TNF- $\alpha$ )-induced apoptotic progression in HeLa cells. HeLa cells were harvested at the indicated time periods after treatment with TNF- $\alpha$ and cycloheximide, and cell extracts were used for (A) immunoblotting using specific antibodies against JNK1, phospho-JNK1, poly-ADP-ribose polymerase (PARP), and $\beta$-actin and (B) measuring caspase-3 activity using Ac-Asp-Glu-Val-Asp-7-amino-4-methylcoumarin as a fluorescent-labeled substrate. The results are representative of at least three independent experiments.

A

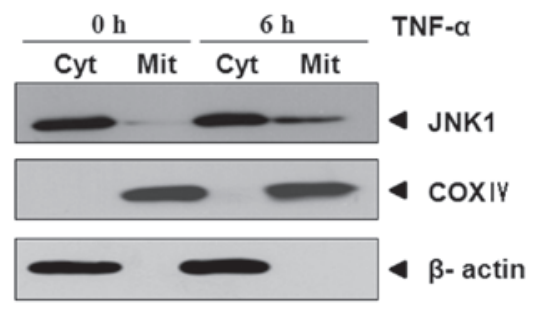

B

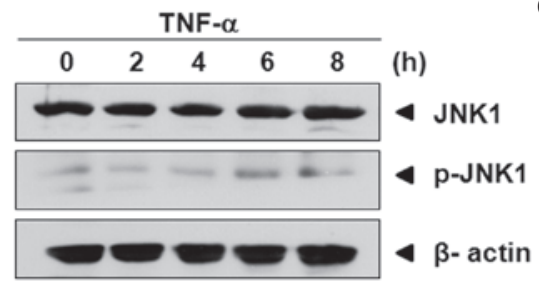

C

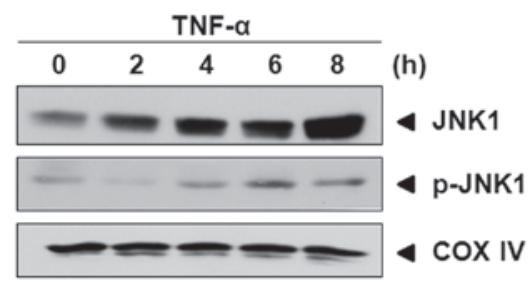

Figure 2. c-Jun N-terminal protein kinase 1 (JNK1) is translocated into the mitochondria of HeLa cells after tumor necrosis factor- $\alpha$ (TNF- $\alpha$ ) treatment. (A) HeLa cells were treated with TNF- $\alpha$ for $6 \mathrm{~h}$ and the cytosolic and mitochondrial fractions were prepared. Each fraction (100 $\mu \mathrm{g}$ protein) was immunoblotted for JNK1, and also for $\beta$-actin and subunit IV of cytochrome oxidase (COX IV) to confirm the clear separation between the cytosolic and mitochondrial fractions. All experiments were performed at least three times. HeLa cells were treated with TNF- $\alpha$ ( $5 \mathrm{ng} / \mathrm{ml}$ ) for indicated time periods and the (B) cytosolic and (C) mitochondrial fractions were prepared. The levels of JNK1 protein and active form of JNK1 (p-JNK1) were determined by using immunoblotting analysis with antibodies against JNK1 or phospho-JNK1.

during TNF- $\alpha$-induced apoptosis, HeLa cells were treated with TNF- $\alpha(5 \mathrm{ng} / \mathrm{ml})$ and cycloheximide $(5 \mu \mathrm{g} / \mathrm{ml})$ for the indicated time periods.

It was determined that JNK1 is phosphorylated at $6 \mathrm{~h}$ and that subsequently the PARP cleavages occur (Fig. 1A). As shown in Fig. 1B, the levels of caspase-3 activity also increased $6 \mathrm{~h}$ after TNF- $\alpha$ treatment. Using these data, it was assumed that activation of JNK1 following TNF- $\alpha$ treatment may be associated with the promotion of apoptosis during TNF- $\alpha$-induced apoptosis.

JNK 1 is translocated from cytosol to mitochondria by TNF- $\alpha$ treatment. It has been reported that JNK mediates the release of Smac after translocation to mitochondria (16). Hence, it was examined whether JNK1 is translocated from cytosol to mitochondria during TNF- $\alpha$-induced apoptosis. As shown in Fig. 2, the levels of JNK1 were increased in mitochondria after $6 \mathrm{~h}$ compared with the levels of JNK1 at $0 \mathrm{~h}$. To confirm this result, cells were treated with TNF- $\alpha$ for $0,2,4,6$ and $8 \mathrm{~h}$ and cytosolic and mitochondrial fractions were prepared. The same levels of JNK1 and a slight increase in the levels of activated JNK1 (p-JNK1) were observed in the cytosol (Fig. 2B). However the levels of JNK1 and p-JNK1 exhibited different patterns of changes in the expression levels of the mitochondria compared with those in the cytosol. Following TNF- $\alpha$ treatment, the levels of JNK1 and p-JNK1 were increased gradually in a time-dependent manner (Fig. 2C).
These results indicate that JNK1 is activated and translocated into the mitochondria during TNF- $\alpha$-induced apoptosis.

JNK1 activity is involved in Smac release from mitochondria during TNF- $\alpha$-induced apoptosis. The present study investigated the role of JNK1 translocation and activation in the release of Smac from mitochondria during TNF- $\alpha$-induced apoptosis. HeLa cells were transfected with a control (mock), JNK1 or DN-JNK1 and then were treated with TNF- $\alpha$ and cycloheximide $24 \mathrm{~h}$ later. Subsequently, subcellular fractionation was performed and the caspase-3 activity levels were measured. It was observed that the release of Smac from mitochondria was promoted by transient expression of JNK1 compared with release in the control, while DN-JNK1 suppressed the release of Smac when compared with that in the control (Fig. 3A). The levels of caspase-3 activity exhibited a similar pattern compared with that of Smac release; activation of caspase-3 increased in HeLa cells overexpressing JNK1 as compared with that of those with mock transfectant, however DN-JNK1 suppressed the activity of caspase-3.

To confirm these results, $24 \mathrm{~h}$ after transfection with mock, JNK1 or DN-JNK1, cells were treated with TNF- $\alpha$ for 0,3 or $6 \mathrm{~h}$ and cytosolic fractions were prepared. Smac release was promoted slightly by transient expression of JNK1 in a time-dependent manner when compared with that in cells transfected with the mock. However, Smac release was 


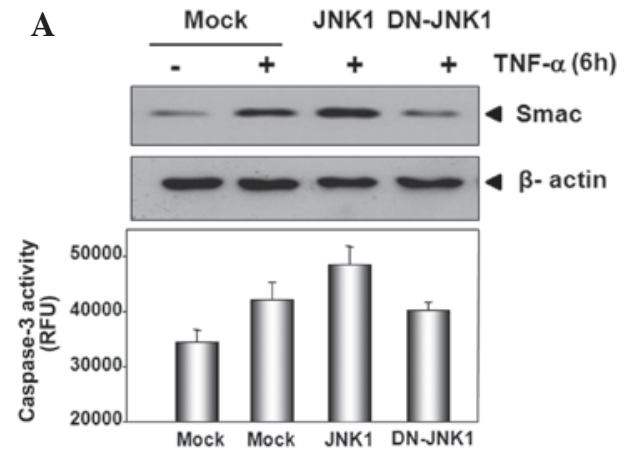

B

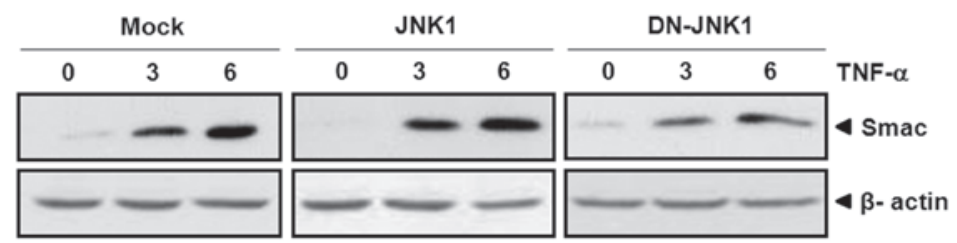

Figure 3. c-Jun N-terminal protein kinase 1 (JNK1) activity is involved in the release of second mitochondria-derived activator of caspases (Smac) from mitochondria during tumor necrosis factor- $\alpha$ (TNF- $\alpha$ )-induced apoptosis. HeLa cells were transfected with an expression vector encoding FLAG-JNK1, the dominant negative version of JNK1 (DN-JNK1), or empty vector (mock). Following transfection, cells were incubated for $24 \mathrm{~h}$ and then treated with (A) TNF- $\alpha$ and cycloheximide for $6 \mathrm{~h}$ or (B) TNF- $\alpha(5 \mathrm{ng} / \mathrm{ml})$ for the indicated time periods. Cells were harvested and subcellular fractionation was performed. To assess the mitochondrial release of Smac, the cytosolic levels of Smac were determined by immunoblotting with anti-Smac antibody. The cell extracts in (A) were also measured for caspase-3 activity using Ac-Asp-Glu-Val-Asp-7-amino-4-methylcoumarin as a fluorescent-labeled substrate.
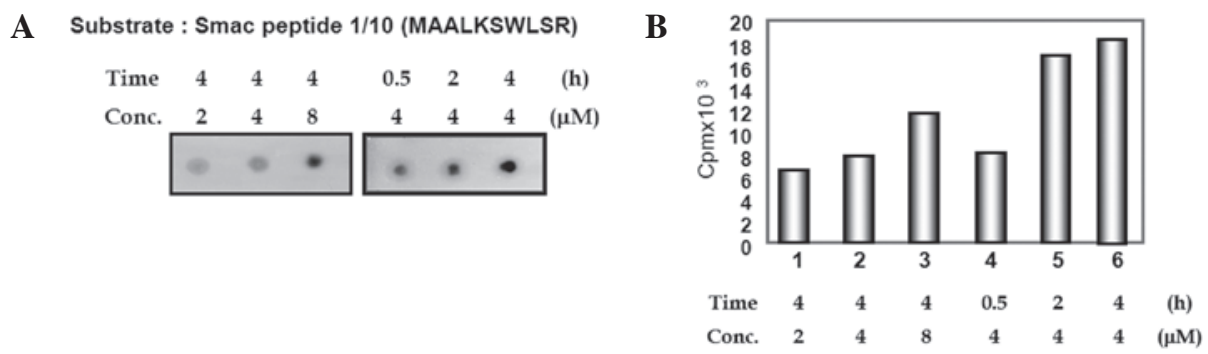

Figure 4. The N-terminus of second mitochondria-derived activator of caspases (Smac) is phosphorylated by c-Jun N-terminal protein kinase 1 (JNK1). The synthetic 10-mer peptide (pep0110) was subjected to in vitro phosphorylation with active JNK1 and the peptide phosphorylation products were immobilized on $\mathrm{p} 81$ phosphocellulose paper and analyzed by (A) autoradiography and (B) liquid scintillation counting.

A

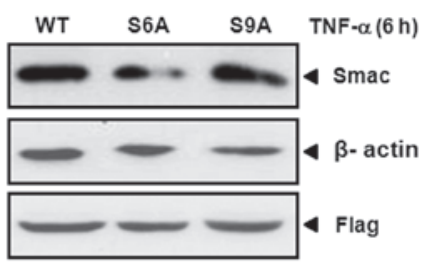

B

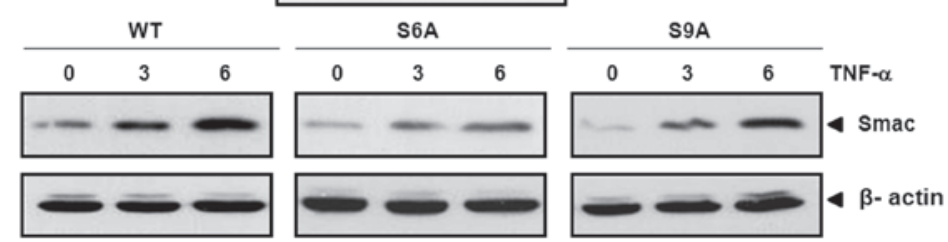

Figure 5. c-Jun N-terminal protein kinase 1 (JNK1)-mediated phosphorylation of second mitochondria-derived activator of caspases (Smac) at the serine 6 residue is involved in the mitochondrial release of Smac during tumor necrosis factor- $\alpha$ (TNF- $\alpha$ )-induced apoptosis. Cells were co-transfected with expression vectors encoding FLAG-JNK1, FLAG-Smac wild-type (WT), FLAG-SmacS6A, or FLAG-SmacS9A. Following transfection, cells were incubated for $24 \mathrm{~h}$ and then treated with (A) TNF- $\alpha$ and cycloheximide for $6 \mathrm{~h}$ or (B) TNF- $\alpha(5 \mathrm{ng} / \mathrm{ml})$ for the indicated time periods. Cells were harvested and subcellular fractionation was performed, and the cytosolic levels of released Smac were subjected to immunoblotting analysis with anti-Smac antibody. The cytosolic levels of $\beta$-actin and FLAG were also determined by immunoblotting as internal controls.

suppressed in HeLa cells transfected with DN-JNK1 compared with that in mock cells. These results suggest that JNK1 has a pro-apoptotic role and that JNK1 activity is involved in Smac release from mitochondria during TNF- $\alpha$-induced apoptosis.

The N-terminus of Smac is phosphorylated by JNK1. It has previously been reported that the phosphorylation of Smac by JNK3 attenuates the apoptotic progression induced by the anticancer drug etoposide (18). Subsequently, the present study investigated the role of Smac phosphorylation in the release of Smac by JNK1. The potential phosphorylation sites of full-length Smac by JNK1 were studied using the public phosphorylation prediction database NetPhos 2.0 server (http://www.cbs.dtu. $\mathrm{dk} /$ services/NetPhos/). When full sequences of Smac were submitted to NetPhos 2.0, several serine and threonine residues were predicted to be phosphorylated by protein kinases. From 
the results of the prediction by NetPhos 2.0, N-terminal serines 6 and 9 of Smac were suggested as putative phosphorylation sites. To examine whether JNK1 could phosphorylate this site, a peptide containing residues of serines 6 and 9 was synthesized; pep0110 corresponded to amino acids 1-10 of full-length Smac. A peptide filter binding assay was performed, which increased the radioactivity of phosphorylated pep0110 in proportion to the quantity of added peptide and the length of time (Fig. 4).

JNK1-mediated phosphorylation of Smac at $N$-terminal serine 6 residue is involved in Smac release during $T N F-\alpha$-induced apoptosis. Experiments were conducted to investigate whether the phosphorylation of Smac at the N-terminus accounts for the effect of JNK1 on Smac. To examine whether the JNK1-mediated phosphorylation of Smac at the serine residues 6 or 9 is associated with the mitochondrial release of Smac, an Smac which could not be phosphorylated by JNK1 was created by substituting serine residues 6 (Smac S6A) and 9 (Smac S9A) with alanine. A cotransfection study was then performed with wild-type (WT) Smac and the mutant versions, Smac S6A and S9A. It was revealed that $\mathrm{Smac}$ release was suppressed by cotransfection of JNK1 with Smac S6A in comparison with other cotransfectants (Fig. 5A). To confirm these results, the same experiments were performed over time. The release of Smac was also revealed to be suppressed by cotransfection of JNK1 with Smac S6A in a time-dependent manner (Fig. 5B).

\section{Discussion}

JNK regulates a number of important cellular events, ranging from gene expression to apoptosis. JNK is activated in response to a variety of extracellular stimuli, including TNF- $\alpha$ (3) and UV (4). The involvement of JNK in TNF- $\alpha$-mediated apoptosis has been debated (8). Sustained activation of JNK may directly promote TNF- $\alpha$-mediated apoptosis (9), however activation of $\mathrm{NF}-\kappa \mathrm{B}$ and JNK together is pivotal in cell proliferation and survival (7). Despite the fact that sustained activation of JNK promotes cell death, the molecular basis of how JNK contributes to TNF- $\alpha$-mediated apoptosis remains to be addressed.

The intrinsic apoptotic pathway is the result of changes in mitochondrial membrane permeability and subsequent release of proapoptotic factors (10). Notably, previous studies have shown that JNK mediates the release of cytochrome $c(15)$ and Smac (16). However, the mechanism of action by which Smac is released from the mitochondria is yet to be determined.

In the present study, it was demonstrated that JNK1 is activated and translocated from cytosol to mitochondria in $\mathrm{HeLa}$ cells and that it can regulate Smac release from mitochondria by phosphorylation of Smac at the N-terminal serine 6 residue during TNF- $\alpha$-induced apoptosis.

Different patterns of change in expression were observed in the intracellular levels of JNK1 and its active phospho-forms using immunoblotting assay. The levels of activated JNK1 were sharply increased at $6 \mathrm{~h}$, followed by PARP cleavage and caspase-3 activation. From these results it was deduced that increased levels of JNK1 activity may be associated with the promotion of apoptosis during TNF- $\alpha$-induced apoptosis.

Subsequently, whether JNK1 is translocated to mitochondria when apoptosis is induced by TNF- $\alpha$ treatment in HeLa cells was investigated and confirmed in the present study.
Chauhan et al (16) have reported that JNK is involved in the release of the mitochondrial protein Smac. The present study examined the role of JNK1 in the promotion of Smac release during TNF- $\alpha$-induced apoptosis. The results revealed that the levels of Smac release were increased by JNK1, but suppressed by DN-JNK1. These results indicate that JNK1 influences TNF- $\alpha$-induced apoptosis by regulating Smac release.

It has previously been reported that phosphorylation of Smac by JNK3 attenuates its interaction with XIAP (18), so the present study investigated whether Smac release by JNK1 is due to phosphorylation. Following prediction analysis using NetPhos 2.0, JNK1 was revealed to phosphorylate Smac peptide0110; a Smac peptide containing residues of serines 6 and 9.

To verify whether the JNK1-mediated phosphorylation of Smac at the serine residues 6 or 9 is involved in the mitochondrial release of Smac, a cotransfection study was performed with Smac WT and the mutant versions, Smac S6A and S9A. It was demonstrated that Smac release was suppressed by cotransfection of JNK1 with SmacS6A in comparison with that of the other cotransfectants. These results indicate that JNK1-mediated phosphorylation of Smac at the N-terminal serine 6 residue is involved in Smac release.

In conclusion, the data presented in this study revealed that the phosphorylation of Smac at serine 6 residue by JNK1 is involved in promoting Smac release from mitochondria. These results suggest that Smac is a major physiological substrate of JNK1 in the regulation of apoptosis, particularly in mitochondria.

\section{Acknowledgements}

This study was supported by the College of Pharmacy-Specialized Research Fund (from the Institute for New Drug Development) of Keimyung University.

\section{References}

1. Chang L and Karin M: Mammalian MAP kinase signalling cascades. Nature 410: 37-40, 2001.

2. Davis RJ: Signal transduction by the JNK group of MAP kinases. Cell 103: 239-252, 2000.

3. Deng Y, Ren X, Yang L, Lin Y and Wu X: A JNK-dependent pathway is required for TNFalpha-induced apoptosis. Cell 115: 61-70, 2003.

4. Tanos T, Marinissen MJ, Leskow FC, et al: Phosphorylation of c-Fos by members of the p38 MAPK family. Role in the AP-1 response to UV light. J Biol Chem 280: 18842-18852, 2005.

5. Weston CR and Davis RJ: The JNK signal transduction pathway. Curr Opin Genet Dev 12: 14-21, 2002.

6. Baud V and Karin M: Signal transduction by tumor necrosis factor and its relatives. Trends Cell Biol 11: 372-377, 2001.

7. Lee SY, Reichlin A, Santana A, Sokol KA, Nussenzweig MC and Choi Y: TRAF2 is essential for JNK but not NF-kappaB activation and regulates lymphocyte proliferation and survival. Immunity 7: 703-713, 1997.

8. De Smaele E, Zazzeroni F, Papa S, et al: Induction of gadd45beta by NF-kappaB downregulates pro-apoptotic JNK signalling. Nature 414: 308-313, 2001.

9. Tang G,Yang J,Minemoto Y andLinA:Blocking caspase-3-mediated proteolysis of IKKbeta suppresses TNF-alpha-induced apoptosis. Mol Cell 8: 1005-1016, 2001

10. Wang X: The expanding role of mitochondria in apoptosis. Genes Dev 15: 2922-2933, 2001.

11. Roy S and Nicholson DW: Cross-Talk in Cell Death Signaling. J Exp Med 192: f21-f26, 2000.

12. Chai J, Du C, Wu JW, Kyin S, Wang X and Shi Y: Structural and biochemical basis of apoptotic activation by Smac/DIABLO. Nature 406: 855-862, 2000. 
13. Wu G, Chai J, Suber TL, et al: Structural basis of IAP recognition by Smac/DIABLO. Nature 408: 1008-1012, 2000.

14. Wilkinson JC, Wilkinson AS, Scott FL, Csomos RA, Salvesen GS and Duckett CS: Neutralization of Smac/Diablo by inhibitors of apoptosis (IAPs). A caspase-independent mechanism for apoptotic inhibition. J Biol Chem 279: 51082-51090, 2004.

15. Tournier C, Hess P, Yang DD, et al: Requirement of JNK for stress-induced activation of the cytochrome c-mediated death pathway. Science 288: 870-874, 2000.
16. Chauhan D, Li G, Hideshima T, et al: JNK-dependent release of mitochondrial protein, Smac, during apoptosis in multiple myeloma (MM) cells. J Biol Chem 278: 17593-17596, 2003.

17. Dérijard B, Hibi M, Wu IH, et al: JNK1: a protein kinase stimulated by UV light and Ha-Ras that binds and phosphorylates the c-Jun activation domain. Cell 76: 1025-1037, 1994.

18. Park BD, Ham YM, Jeong HJ, et al: Phosphorylation of Smac by JNK3 attenuates its interaction with XIAP. Biochem Biophys Res Commun 361: 994-999, 2007. 